\title{
Synethic or Biochemical Material for Reconstruction of Pelvic Organ Prolapse, It Matters!
}

\author{
Wei-Jiun Li ${ }^{1}$, Lee-Wen Huang ${ }^{1,2}$, Ma-Lee Ko, Jier-Zen Chang ${ }^{1,2}$, \\ Hung-Chi Chang ${ }^{3}$, Pincheng $\mathrm{Pan}^{4}$, Pintong $\mathrm{Pan}^{4}$ and Hun-Shan \\ $\operatorname{Pan}^{1,2 *}$
}

\author{
Institutional affiliations \\ ${ }^{1}$ Department of Obstetrics and Gynecology, Shin-Kong Hospital, Taipei, \\ Taiwan. \\ ${ }^{2}$ College of Medicine, Fu-Jen Catholic University, New Taipei, Taiwan. \\ ${ }^{3}$ Hungchi Woman \& Children's Hospital. \\ ${ }^{4}$ Science Department, University of British Columbia, Canada.
}

\section{Corresponding author}

Hun-Shan Pan,

Department of Obstetrics and Gynecology,

Shin-Kong Hospital, No 95, Wen-Chang Road, Suih Lin District, Taipei, Taiwan.

Tel: +886-(0)921-068-150;

Fax: +886-(0)2-2838 9335;

E-mail: pan5511@me.com

Received Date: August 23, 2020

Accepted Date: August 25, 2020

Published Date: Sep 04, 2020

\section{Abstract}

Pelvic Organ Prolapse (POP) is a disabling and chronic condition that affects women of all ages. Pelvic organs can protrude outside the body through the vagina due to weakness in the pelvic floor. POP affects patients both physically and psychologically. Most women with POP suffer from at least one other pelvic floor disorder, such as incontinence. Pelvic organ prolapse increases nationwide, namely, anterior, posterior and middle compartments prolapse. Plenty of different kits had created in recent years for reconstruction surgery. Among those surgical kits, synthetic material augmentation plays a major role, for last Decade, however, Food and Drug Administ-ration raised (FDA) a red flag due to massive complication, such as spontaneous vaginal pain, extrution, there-fore, Nowdays, selection of proper material for reconstruction surgery is important for life quality. it divides into two systems: anchor non-anchor system. Hence, we attempt to compare a novel modified surgical technique involving a non-anchor system with the conventional anchor approach to determine the effectiveness of these two systems for treating POP. In this study, we compared the benefit, disadvantage, side effects, and complications for the two systems. Then we found that the modified tiling approach can be used to prevent the aforementioned surgical risks. Furthermore, if patients cannot bear the cost of using the required instruments, they can consider undergoing the modified nonanchor surgical procedure.

2. Keywords: Cystocele; Enterocele; Incontinence; Pelvic Organ Prolapse; Rectocele; Uterine Prolapse

\section{Introduction}

Pelvic Organ Prolapse (POP) is a common condition that many adult women experience and are too embarrassed to talk about. However, the improvement in quality of life and extension of average life expectancy has influenced women's daily lifestyle, causing POP to gradually receive attention from medical experts. Because pelvic muscle and fascia weaken over time, POP causes inconvenience daily living activities. POP commonly occurs in the anterior, medial, and posterior compartment of the pelvis and may occur in more than one place at the same time. Studies on the knowledge and method of pelvic organ reconstruction have typically focused on using anchor and non-anchor systems.

A demographic study revealed that the number of women aged over 80 years in the United States and Canada is increasing rapidly, with the number of elderly women expected to continue to increase by 2050 [1]. If the current female generation continues to maintain an active lifestyle until old age, then the number of women seeking medical attention for prolapse problems will increase. Symptoms of pelvic organ prolapse differ among people. According to clinical guidelines for female pelvic organ and urinary incontinence published by medical associations, POP symptoms may be unperceivable, whereas some people may experience the following symptoms: protrusion from the vagina, difficulty engaging in sexual intercourse, and a mixture of symptoms in the urinary tract such as urination urgency, frequent urination, urinary incontinence, and voiding or defecatory difficulties. These symptoms are mostly related to prolapse in the anterior and posterior compartment of the pelvis in the vagina [2]. Risk factors of POP include ethnicity, pregnancy, vaginal delivery, abnormal anatomical structures, abnormal collagen composition, vaginal removal due to prolapse, or radical surgery for cancer. Promoters of POP include obesity, old age, chronic pulmonary disease, myopathy, neuropathy, constipation, and menopause. Patients who are less than 60 years old and $65 \mathrm{~kg}$ are at high risk of experiencing recurrent POP after surgery. A study estimated that the number of patients requiring treatment for pelvic disease will double in the next 30 years, and that approximately 300,000 people would need to receive surgery for pelvic reconstruction or urinary incontinence every year [1]. Nevertheless, patients needing surgical treatments account for only a portion of POP patients, because some patients choose to treat their POP problems conservatively whereas others believe that POP is a result of aging and are thus too embarrassed to talk about it and choose to not seek for medical assistance. Therefore, future gynecologists must endeavor to develop a reconstruction treatment that is safe, yields high success rate, incurs low level of postoperative pain, and minimizes patients' medical costs. However, the medical costs generated from using most of the anchor systems for POP treatment are not covered by the Taiwan's National Health Insurance (NHI) system. To address the adaptation and benefit problems for certain patients, and to determine the effectiveness of these two systems for treating POP, this study compares a novel modified non-anchor surgical technique with the conventional anchor approach.

\section{Materials and Methods}

From January 2018 to January 2020, patients diagnosed with prolapse in the anterior compartment of the pelvis to the vaginal opening of the cervix are patients with apical prolapse. According to urodynamic tests, 175 patients were found to have obstructive urinary incontinence. Excluding 23 patients who are unfit for surgery as diagnosed by anesthesiologists, 152 patients were enrolled in this study. Of the recruited patients, 81 and 71 patients respectively underwent pelvic reconstruction surgery and Tension-free Vaginal Tape-Obturator (TVT-O) surgery using synethic mesh commercial kit (uphold) and a modified non-anchor system. The procedure for the modified non-anchor surgery involved developing an assistive suspension system using sarco-spinal suspension for level one and Biochemical material which called PLA masters ${ }^{\circledR}$ (polylacticacid $5 \mathrm{~mm} * 10 \mathrm{~mm} * 130 \mathrm{~mm}$ ) applied Bilateral para-vaginal space) modifying for fixating anterior compartment it in the vagina (Figure 1). 


\section{Journal of Clinical Obstetrics and Gynecology Research}
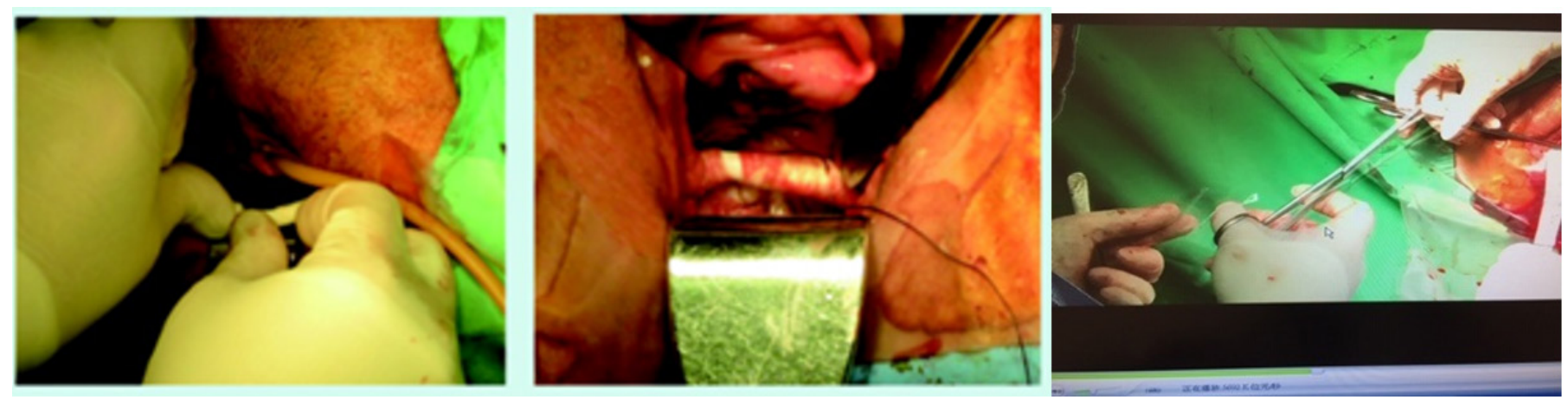

Figure 1: The modified kit inserts in vagina to support nonachor PLA.

Sling surgery, which is covered in Taiwan's NHI system, involves using ring double hooks left over from a suspension surgery. The procedure of a sling surgery was as follows: take a ring-shaped plastic double-pointy tip of the hooks and cut open one of the hollow parts such that the tip of the ring hook is inserted into the opened hollow part; then, according to the width of the patient's vagina and location of the sagging part, adjust the place where the ring object is inserted. Generally, the ring object is placed in between the bladder and cervix and then stitched to the two sides of the anterior fornix near the two sides of the vagina. After three to four weeks, the ring object was removed, and PLA was used and cut in a way that the area of its base is $2 \times 3 \mathrm{~cm}$ with two ends about $7 \mathrm{~cm}$ long, forming an inverted-V mesh. The mesh was placed $1-2 \mathrm{~cm}$ from the upper end of the cervix and stitched to the cervix; the two ends of the mesh arm were inserted into the paravaginal space and then fixated with the ring object for one weeks. Subsequently, the ring object was extracted during the next examination.

The two groups of patients were followed up 3 months, 6 months, and 1 year after the surgery starting from the postoperative examination. Furthermore, a month after the surgery, three sessions of rehabilitation treatment involving electrical stimulation were arranged, with each session containing 18 types of rehabilitation activity and administered 1 month, 3 months, and 6 months after surgery. The prognoses of the two types of surgical technique were compared. Data were statistically analyzed using a t test with the confidence interval set at $95 \%$.

\section{Results}

The two groups of patients were followed up 3 months, 6 months, and 1 year after the surgery starting from the postoperative examination. Furthermore, a month after the surgery, three sessions of rehabilitation treatment involving electrical stimulation were arranged, with each session containing 18 types of rehabilitation activity and administered 1 month, 3 months, and 6 months after surgery. Each rehabilitation session lasted 20 minutes. In addition, patients were required to return every month for an internal check-up, and subsequently received magnetic rehabilitation treatment 1 month, 3 months, and 6 months after surgery. The prognosis of the two surgical techniques after 1 year was compared, focusing on the level of pain during sexual intercourse, degree of recovery, the probability of recurrent prolapse, and infection rate. The results (Table 1) indicated the erosion rate ( $8.3 \%$ vs. $1.7 \%)$ and the success rate $(95.3 \%$ vs. $92.1 \%)$ of the two groups and that both groups differed primarily with regards to dyspareunia ( $33 \%$ vs. $1.2 \%$ ), pain in the vaginal or buttock area $(23.2 \%$ vs. $1.6 \%)$, and hematoma $(4.5 \%$ vs. $2.5 \%)$. No significant difference was observed regarding other prognoses, including vesicorectal injury, urinary tract infection (17.3\% vs. 19.1\%), urinary retention, recurrent urinary incontinence, and recurrent prolapse.

\begin{tabular}{|l|l|l|}
\hline & $\begin{array}{l}\text { Anchor } \\
\text { System }\end{array}$ & $\begin{array}{l}\text { Modified Non- } \\
\text { Anchor System } \\
\text { (PLA) }\end{array}$ \\
\hline Numbers & 81 & 71 \\
\hline Success rate & $95.30 \%$ & $92.10 \%$ \\
\hline Erosion Rate & $8.30 \%$ & $1.70 \%$ \\
\hline Dyspareunia & $33 \%$ & $1.2 \%^{*}$ \\
\hline $\begin{array}{l}\text { Pain in the Vaginal } \\
\text { or Buttock Area }\end{array}$ & $23.20 \%$ & $1.6 \%^{*}$ \\
\hline Hematoma & $4.50 \%$ & $2.5 \%^{*}$ \\
\hline
\end{tabular}

\section{Discussion}

POP is the protrusion of the pelvic organ from the vagina, and more than half of women aged over 50 years are diagnosed with this condition. The prevalence of lifetime POP is approximately 30\%-50\% [1]. Previously, most postpartum women do not rest properly after delivery because of living and work-related needs; they often begin engaging in labor early, thus increasing the occurrence rate of POP. Although POP is not life threatening, patients may experience disturbing symptoms at any time, which severely influences their quality of life.

POP symptoms vary across different people. During the early stages of POP, patients do not show symptoms; however, increase in abdominal pressure for prolonged period induces a bulging sensation in the lower abdomen, as well as abdominal pain and lower backache. However, when abdominal pressure increases suddenly (e.g., coughing, sneezing, and jumping), it causes genital prolapse [3]. Women with POP at first frequently complain about feeling the presence of foreign objects inside their vagina or sensations of swelling and a sense of heaviness in the pelvis as if something has fallen off. Patients with uterine prolapse may feel a lump in the lower part of their vagina, whereas patients with cystocele may complain that they feel as if they are sitting on a ball [4]. Such feelings are mild in the morning and intense at night but are alleviated once they lie down or rest for some time. Urinary incontinence and POP are a comprehensive presentation of relaxation in the pelvic muscle; they may occur simultaneously, or occur consecutively one after the other. Some people may also experience defecatory difficulty. Risk factors of POP include ethnicity, pregnancy, vaginal delivery, abnormal anatomical structures, abnormal collagen composition, vaginal removal due to prolapse, or radical surgery for cancer. Promoters of POP include obesity, old age, chronic pulmonary disease, myopathy, neuropathy, constipation, and menopause. Patients who are less than 60 years old and $65 \mathrm{~kg}$ are at 
high risk of experiencing postoperative recurrent POP [2]. According to Olsen (1997), women with an average life expectancy of 79 years have approximately $11 \%-12 \%$ chance of receiving at least one surgery for POP or urinary incontinence, and a $29.2 \%$ chance of undergoing surgery again for these two conditions [5]. If the current female generation continues to maintain an active lifestyle until their old age, then the number of women seeking medical attention for prolapse problems would increase. A study estimated that the number of patients requiring treatment for pelvic diseases will double in the next 30 years, [6] and that approximately 300,000 people would need to receive surgery for pelvic reconstruction or urinary incontinence every year [7]. Nevertheless, patients needing surgical treatments account for only a portion of POP patients, because some patients choose to treat their POP problems conservatively whereas others believe that POP is a result of aging and thus are too embarrassed to talk about it and choose to not seek for medical assistance [8].

Modified non-anchor systems are an alternative treatment for women with pelvic sagging and urinary incontinence, particular those in the middle class and below. However, because the follow-up period in the present study was brief, more time is needed in the future to observe subsequent changes. Postoperative education is also a focus; patients should be informed not to pick up heavy objects after a surgical operation. Patients with internal disease such as chronic obstructive pulmonary disease should be informed of risks that influence the success rate of a surgery [9]. Vaginal Support Device (VSD) must be inserted into the vagina and fixated at the intersection of the bladder and the vagina for at least one weeks [10]. Generally, absorbable vicryl suture 3-0 is used to fix the VSD at the intersection; after the VSD has been removed, premarin must be applied to the vagina. In this study, the two groups of patients were required to apply premarin for three months, after which they underwent three sessions of electrical stimulation rehabilitation (each session involves 18 activities). The rehabilitation was aimed at maintaining the postoperative effect, follow-up on patients' prognostic conditions, and provides intervention measures in a timely manner. This study found that the urinary tract infection ratios of the two groups were higher than those reported in [11]. This difference may be attributable to the duration of urinary catheter placement, which was approximately three days in the present study. Therefore, shortening the duration of urinary catheter placement should reduce the probability of an infection. The main prognosis was an improvement in the level of perceived pain; because the modified surgical technique requires no puncture into the fascia muscle and because the tape used is fixated using a self-healing adhesion approach, such surgical procedure does not elicit pain when walking or sitting on the buttock [12]. Regarding the method for resolving hematoma, the author does not recommend removing the mesh. Instead, antibodies should be administered to actively prevent infection and the level of hemoglobin should be monitored in order to transfuse blood when necessary. Thus, severe hematoma can be eliminated gradually. In the present study, patients with postoperative hematoma were treated conservatively and did not need surgery again. Because some of the patients in our case study are still undergoing follow-up examinations, conducting more case studies may facilitate reducing the errors generated to an acceptable range. Concerning mesh exposure, the FDA recommended doctors to receive specialized training and advised them to be cautious when inserting needles to prevent injuries in the intestine, bladder, and blood vessels [13]. Before a surgery, it is best to inform the patient that the mesh is placed permanently in the vagina and that even surgeries may be ineffective for treating the complications arising thereof. Regarding technical aspects, when placing the anterior mesh, surgeons must pay attention to bladder perforation. In addition, some surgeons are accustomed to performing perforation from the pubic bone next to the obturator [14] and then let the mesh slip down naturally to the obturator. This approach is slightly risky because it may perforate the branches of the obturator vessels. The authors recommend that when the trocar enters the anterior vaginal compartment, surgeons should compress the trocar slightly toward the rear end and then perforate it into the obturator muscle. Subsequently, when the trocar is inserted into the vagina, the best practice is to touch both sides of the lateral sulcus in the vagina. Occasionally, the trocar may perforate the vaginal wall [15]. Thus, when stitching the wound in the vagina without checking it beforehand, the mesh may have been displaced already, which would be problematic. When inserting the posterior mesh, attention must be paid to the Alcock's canal behind the ischial spine because the internal pudendal artery is connected to the nerve [16]. Therefore, care should be taken when inserting the mesh at this area. Overall, the modified tiling approach can be used to prevent the aforementioned surgical risks. Furthermore, if patients cannot bear the cost of using the required instruments, they can consider undergoing the modified non-anchor surgical procedure.

\section{References}

1. Nieminen K, Hiltunen R, Takala T, Kirsti N, Eila H, Mauri M, et al. Outcomes after anterior vaginal wall repair with mesh:a randomized, controlled trial with a 3 year follow-up. Am J Obstet Gynecol. 2010;203(3):235.e1-8.

2. Lopes ED, Lemos NL and Carramão Sda S. Transvaginal polypropylene mesh versus sacrospinous ligament fixation for the treatment of uterine prolapse: 1-year follow-up of a randomized controlled trial. Int Urogynecol J. 2010;21(4):38994.

3. Iglesia CB, Sokol AI, Sokol ER, Bela IK, Robert EG, Susan S, et al. Vaginal mesh for prolapse: a randomized controlled trial. Obstet Gynecol. 2010;116(2 Pt 1):293-303.

4. Maher CF, Feiner B, DeCuyper EM, Cathy JN, Kacey VH and Peter OR. Laparoscopic sacral colpopexy versus total vaginal mesh for vaginal vault prolapse: a randomized trial. Am J Obstet Gynecol. 2011;204(4):360.el-7.

5. Altman D, Vayrynen T, Engh ME, Susanne A, Christian F and Nordic TMG. Anterior colporrhaphy versus transvaginal mesh for pelvic-organ prolapse. N Engl J Med. 2011;364(19):182636.

6. Olsen AL, Smith VJ, Bergstrom JO, Colling JC and Clark AL. Epidemiology of surgically managed pelvic organ prolapse and urinary incontinence. Obstet Gynecol. 1997;89(4):501-6.

7. Bush MB, Liedl B, Wagenlehner F, Yassouridis A and Petros PE. Effects of posture and squatting on the dynamics of micturition. Int Urogynecol J. 2015;26(5):779-80.

8. Norton P, Baker J, Sharp H, et al. Genito-urinary prolapse: Relationship with joint mobility. Neuro Urodyn. 1990;9:321322.

9. Baden WF and Walker T. Surgical Repair of Vaginal Defects. Philadelphia, Pa: Lippincott Williams \& Wilkins; 1992.

10. Samuelsson EC, Arne Victor FT, Tibblin G and Kurt FS. Signs of genital prolapse in a Swedish population of women 20 to 59 years of age and possible related factors. Am J Obstet Gynecol. 1999;180(2 Pt 1):299-305.

11. Jirschele K, Seitz M, Zhou Y, P Rosenblatt, P Culligan, and $\mathrm{P}$ Sand. A multicenter, prospective trial to evaluate meshaugmented sacrospinous hysteropexy for uterovaginal prolapse. Int Urogynecol J. 2015;26(5):743-8.

12. Lazarou G, Scotti RJ, Zhou HS, Magdy SM and Powers K. Preoperative Prolapse Reduction Testing as a Predictor of Cure 


\section{Journal of Clinical Obstetrics and Gynecology Research}

of Urinary Retention in Patients with Symptomatic Anterior Wall Prolapse. Int Urogynecol J Pelvic Floor Dysfunct. 2004;15(3):175-8.

13. Rane A1 and Iyer J. Posture and micturition: does it really matter how a woman sits on the toilet? Int Urogynecol J. 2014;25(8):1015-21.

14. Bump RC, Mattiasson A, Bo K, L P Brubaker, J O DeLancey, $\mathrm{P}$ Klarskov, et al. The standardization of terminology of female pelvic organ prolapse and pelvic floor dysfunction. Am J Obstet Gynecol. 1996;175(1):10-7.
15. Scotti RJ, Flora R, Greston WM, L Budnick and J HutchinsonColas. Characterizing and reporting pelvic floor defects: the revised New York classification system. Int Urogynecol J Pelvic Floor Dysfunct. 2000;11(1):48-60.

16. Nilsson CG. Creating a gold standard surgical procedure: the development and implementation of TVT. Int Urogynecol J. 2015;26(4):467-9. 